\title{
Hospital accreditation and clinical nutrition service in South Korea: a comment on Um et al.'s paper
}

\author{
Rui Poínhos ${ }^{1}$, Flora Correia ${ }^{1,2,3}$ \\ ${ }^{1}$ Faculdade de Ciências da Nutrição e Alimentação, Universidade do Porto, Rua Dr. Roberto Frias, Porto, Portugal; ${ }^{2}$ Centro Hospitalar de São João, \\ E.P.E., Alameda Prof. Hernâni Monteiro, Porto, Portugal; ${ }^{3}$ Unidade de Investigação e Desenvolvimento de Nefrologia, Faculdade de Medicina, \\ Universidade do Porto, Alameda Prof. Hernâni Monteiro, Porto, Portugal \\ Correspondence to: Rui Poínhos. Faculdade de Ciências da Nutrição e Alimentação, Universidade do Porto, Rua Dr. Roberto Frias, 4200-465, Porto, \\ Portugal. Email address: ruipoinhos@fcna.up.pt. \\ Comment on: Um MH, Lyu ES, Lee SM, et al. International hospital accreditation and clinical nutrition service in acute care hospitals in South Korea: \\ results of a nationwide cross-sectional survey. Asia Pac J Clin Nutr 2018;27:158-66.
}

Received: 23 April 2019. Accepted: 30 April 2019; published: 24 May 2019.

doi: $10.21037 /$ jhmhp.2019.05.01

View this article at: http://dx.doi.org/10.21037/jhmhp.2019.05.01

The accreditation systems in health care institutions are one of the most relevant current health-related topics. It is widely recognized as a major step towards the implementation and maintenance of good practices; this needs, however, that both changes and their maintenance are adequately and frequently assessed. Moreover, and as most health care institutions provide health care in different areas, these assessments should not only be global, but also focus specific areas of intervention. At this level, the paper "International hospital accreditation and clinical nutrition service in acute care hospitals in South Korea: results of a nationwide cross-sectional survey" (1) is of great interest, as it explores the differences in clinical nutrition services between Joint Commission International (JCI) accredited and nonaccredited acute care hospitals. We positively highlight the scarce literature focusing such a specific theme.

Despite the overall interest of the paper, some weaknesses should, however, be considered. Perhaps the most immediate regards that, despite the high participation rate $(81.4 \%$, corresponding to 35 of all 43 acute care hospitals in South Korea), there is an important lack of information regarding which hospitals did not participate. The information on the participation rates in the two groups separately would provide a better setting to discuss some of the results, concerning possible bias associated to different participation rates and its underlying motives. This limitation overlaps the one referred by the authors concerning the small sample size of accredited hospitals. Concerning this, it is also worth noticing that the authors cannot claim that their research "was the first to survey the overall state of clinical nutrition services in the 43 acute care hospitals in South Korea", since only data from 35 of these were analyzed.

Also, the authors tend to describe the results in a way that favors the results in accredited acute care hospitals. For instance, while the higher rate of malnutrition cases and subsequent intervention in non-accredited hospitals is deeply discussed despite statistically non-significant, the number of patients/day is clearly described as showing a "significant difference" between the two groups. Results without significant differences are also often described simply as "regardless of JCI accreditation". We believe that a more neutral description and interpretation of the results would benefit the readers, as well as would facilitate its use to improve practices concerning these health care services.

Also concerning nutrition screening, some results should also be highlighted. The absence of significant differences between accredited and non-accredited hospitals regarding nutrition screening rate should not be expected or, at least, the nutrition screening rate should be of $100 \%$ in JCI accredited hospitals, despite statistical significance. The same is applicable to monitoring and reassessment, not performed in two of the eight accredited hospitals evaluated. Patients in acute care hospitals should be considered, a priori, at risk of malnutrition, and it is widely recognized that this risk increases throughout hospitalization. Thus, the universal nutrition screening, monitoring and reassessment should be the target, especially in hospitals which received 
accreditation. This seems feasible, given that the authors report results from previous research indicating that " $100 \%$ of acute care hospitals were equipped with computerized nutritional screening systems for timely nutrition screening of inpatients to allow for quick nutrition evaluations".

Besides what concerns our previous considerations regarding the increased risk of malnutrition during the hospitalization period, it is unclear why some results concerning inpatients $v s$. outpatients seem to be interpreted differently. As referred in the discussion, the focus on inpatient care is "considered important for FCI accreditation, rather than on outpatient nutrition counseling, which is more profitable". However, and although a benefit-cost analysis is fundamental to the adequate functioning of any health care system, we do not agree that profit should have such a weight when considering the quality of health care services.

Nevertheless, this study clearly reveals that accredited acute care hospitals in South Korea present an overall better clinical nutrition service than those without JCI accreditation. Contrary to what we referred regarding the description of some results, the discussion focuses a very pertinent issue which may bias the results: "...since both $\mathcal{F} C I$ and KOIHA reaccredit hospitals every 3 years, some hospitals might perform medical services just to pass the accreditation evaluation process". This possibility leads us to think that, besides the authors' final suggestion that hospitals should, among other improvements, establish continuous followup programs until reaccreditation, the somehow scheduled evaluation prior to reaccreditation should be rethought.

The results concerning clinical dietitians' job satisfaction level indicate the need for future improvements. Hospital

doi: 10.21037/jhmhp.2019.05.01

Cite this article as: Poínhos R, Correia F. Hospital accreditation and clinical nutrition service in South Korea: a comment on Um et al.'s paper. J Hosp Manag Health Policy 2019;3:11. accreditation may lead to higher rationalization of resources, and this would be expected to increase job satisfaction. However, as the authors discuss, some job satisfaction items presented lower scores in accredited hospitals, being a possible explanation the higher proportion of temporary clinical dietitians. The lower satisfaction regarding salary and job stability may limit long-term improvements in clinical practice.

To conclude, we consider that the article (1) provides relevant information on both the effects and procedures related to the accreditation of acute care hospitals in South Korea regarding clinical nutrition services. Further studies should focus on the features in which the accreditation didn't show to make a great difference, in order to improve the overall clinical nutrition practice and to maximize the utility of accreditation.

\section{Acknowledgements}

None.

\section{Footnote}

Conflicts of Interest: The authors have no conflicts of interest to declare.

\section{References}

1. Um MH, Lyu ES, Lee SM, et al. International hospital accreditation and clinical nutrition service in acute care hospitals in South Korea: results of a nationwide crosssectional survey. Asia Pac J Clin Nutr 2018;27:158-66. 